\title{
Imperfective Readings: Partitions as Quantificational Domains
}

\author{
Ashwini Deo \\ Yale University
}

\section{Introduction}

In languages which realize the imperfective aspect (IMPF), sentences containing IMPF-marked verbs exhibit at least three distinct readings (e.g. Italian (Romance), Gujarati (Indo-Aryan), Arabic (Semitic, Ryding 2005), and Bambara (Mande, Tröbs 2004). ${ }^{1}$ These are the progressive or event-in-progress reading (with lexically episodic predicates), the habitual/generic or characterizing reading (with lexically episodic predicates) and the continuous reading (with lexically stative predicates). The correlation of IMPF-marking with these three readings has been observed to be a robust cross-linguistic tendency (Comrie 1976, Dahl 1985, Bybee et al. 1994, Deo 2006 and others) that suggests a single semantic core from which the three readings might arise. These readings are illustrated in (1) with examples from Gujarati. ${ }^{2}$

a. niśā rasoḍā-mā roṭli banāv-e ch-e

N.NOM kitchen-LOC bread.NOM make-IMPF.3.SG PRES-3.SG

'Nišā is making bread in the kitchen (right now).' event-in-progress

b. niśā roṭli banāv-e ch-e

N.NOM bread.NOM make-IMPF.3.SG PRES-3.SG

'Niśā makes bread (habitually).'

characterizing

c. niśā navsāri-mā rah-e ch-e

N.NOM.SG Navsari-LOC live-IMPF.3.SG PRES-3.SG

Niśā lives in Navsari.

continuous

I am grateful to David Beaver, Andrea Bonomi, Ariel Cohen, Cleo Condoravdi, Veneeta Dayal, Fabio Del Prete, Itamar Francez, Paul Kiparsky, Larry Horn, Mokshay Madiman, Chris Pinõn, Judith Tonhauser, Zoltan Szabó and the audiences at SALT XIX, Workshop on Imperfective Form and Imperfective Meaning at Yale, and the Genericity Conference for comments and discussion. The usual disclaimers apply.

${ }^{1}$ The abbreviations IMPF and PROG by themselves refer to the semantic categories of the imperfective and the progressive respectively, while the morphological marking that realizes these categories will be called IMPF-marking or PROG-marking. Following Comrie (1976), languagespecific aspectual markers will be written with capitalized initials (e.g. the English Progressive or the Gujarati Imperfective).

${ }^{2}$ Throughout this paper, I use the Gujarati Imperfective as an instance of the realization of IMPF. The data have been collected from native speakers of standard Gujarati from Navsari living in South Gujarat. 
Progressive (PROG)-marking, on the other hand, most saliently exhibits the event-in-progress reading (e.g. English, Italian, Hindi).

(2) Mary was biking to work... when she got hit by a bus. event-in-progress

The goal of this paper is to answer two questions raised by these crosslinguistically attested patterns. First, how can the event-in-progress, characterizing, and continuous readings be derived from a single semantic core associated with IMPF? Second, what is the source of the typological variation in the manifestation of imperfectivity, viz. the semantically narrow PROG (as in English) and the more general IMPF (as in Gujarati)?

Building on the idea that IMPF involves universal quantification, this paper proposes that the domain of this quantifier is a regular partition (i.e. a set of collectively exhaustive, non-overlapping, equimeasured subsets) of an interval. The partition-measure (the length of each partition-member) is a contextually determined variable. PROG differs from IMPF in that its partition-measure is restricted to measures of infinitesimal value rather than being contextually determined. While this proposal allows us to account for the event-in-progress reading of PROG, it is not able to capture some other facts about the distribution of PROG - viz. unacceptability of PROG-marking with stative predicates and the characterizing reading available to PROG-marked sentences containing lexically episodic predicates.

\section{IMPF-as-Universal Accounts}

'IMPF-as-universal' is my term for the set of accounts in which imperfective meaning is analyzed as involving a strong, quasi-universal quantifier (e.g Newton 1979; Bonomi 1997; Lenci \& Bertinetto 2000; Cipria \& Roberts 2000). The characterizing readings of IMPF-marked sentences are attributed to the presence of this quantifier, whose domain is taken to be contextually 'relevant' or 'characteristic' instances (i.e intervals, events, or situations). This analysis locates the quantificational force associated with covert operators GEN or HAB in an overt piece of morphology, thus systematizing the relation between linguistic form (IMPF-marking) and linguistic meaning (characterizing sense). Despite its attractiveness and simplicity however, the IMPF-as-universal analysis suffers from some shortcomings.

\subsection{Non-explicit Restriction}

First, the precise role of context in identifying the restriction of the quantifier is not explicated. The task is especially complicated when IMPF-marked sentences are uttered with neutral intonation out of the blue. Bare habitual sentences without explicit domain restrictors, such as (1b) (repeated in (3a)), best illustrate this problem. 
Adapting from Bonomi (1997) (with some notational variants), (3a) can be said to have the logical form in (3b), which says that every contextually relevant subinterval $i^{\prime}$ of the reference interval $i$, which includes now, overlaps with (the time of) an event $e$ of Niśā making bread. ${ }^{3}$

a. niśā roṭli banāv-e ch-e

N.NOM bread.NOM make-IMPF.3.SG PRES-3.SG

'Niśā makes bread.'

b. $\exists i\left[\right.$ now $\subseteq i \wedge \forall i^{\prime}\left[i^{\prime} \subseteq i \wedge \underline{\operatorname{Cont}\left(i^{\prime}\right)} \rightarrow \exists e\left[\right.\right.$ Niśāa-make-bread $\left.\left.\left.(e) \wedge \tau(e) \circ i^{\prime}\right]\right]\right]$

If (3a) is uttered with neutral intonation, it is not clear what the Contextually relevant set of intervals that is being quantified over by IMPF is. It could possibly be the set of intervals in which Niśā cooks. The sentence then conveys the proposition that whenever Niśā cooks, she makes bread. This is too strong, since the sentence may be judged true despite several cooking occasions that do not involve bread-making.

In these kind of cases, then, restricting the domain to relevant or characteristic intervals/situations amounts to treating contextual relevance as a predicate in its own right $\left(\lambda_{i} \operatorname{Cont}(i)\right)$, rather than assuming a pragmatic mechanism for recovering the domain of quantification from the context. This greatly undermines the value of the IMPF-as-universal analysis and requires an ad hoc weakening of the universal quantifier to account for the weaker truth-conditions.

A related problem not discussed here is that the IMPF-as-universal analysis breaks down in the presence of overt Q-adverbs that do not have coinciding quantificational force (e.g. seldom, often), because it is not clear how to tease apart the contribution of the two quantifiers (see Deo 2009a, b for an explication of this problem).

\subsection{Exception Tolerance}

A further, more serious problem arises in dealing with the exception-tolerating behavior of characterizing sentences where the domain is explicitly restricted. In (4), sānjhe 'in the evening' provides the restriction for the universal quantifier but the sentence is judged true even if there are some evenings within the reference interval in which Niśā does not make bread.

a. Niśā sānjhe roṭli banāv-e ch-e

N.NOM evening.LOC bread.NOM make-IMPF.3.SG PRES-3.SG

'Niśā makes bread in the evening.'

b. . $\exists i\left[\right.$ now $\subseteq i \wedge \forall i^{\prime}\left[i^{\prime} \subseteq i \wedge \operatorname{evening}\left(i^{\prime}\right) \rightarrow \exists e[\right.$ Niśā-make-bread $(e) \wedge \tau(e) \circ$ $\left.\left.\left.i^{\prime}\right]\right]\right]$

\footnotetext{
${ }^{3}$ Bonomi's analysis applies to the Italian Imperfetto, which also realizes the past tense.
} 
The salient reading of (4) is that there is a regular distribution of breadmaking events in the evening by Niśā within the reference interval, not that every evening contains such an event. An IMPF-as-universal account that seeks to replace GEN cannot easily tackle the exception tolerating behavior of IMPF-marked characterizing sentences. ${ }^{4}$

\subsection{The Intensional Component}

IMPF-marked characterizing sentences describe generalizations that are expected to hold indefinitely across time (Lenci \& Bertinetto 2000; Menendez-Benito 2002). Further, they may be judged true despite the absence of verifying instances. For instance, (5) is considered true if uttered at the inauguration of a new institution for the blind despite no actual events of helping blind people, as long as the institution aims to do so (compare: Members of this club help each other in emergencies.)

$\bar{a}$ sansthā andhā māṇsā-ne madad kar-e ch-e This institution.NOM blind.PL people.PL-DAT help do-IMPF PRES-3.SG

'This institution helps blind people.'

What is relevant in such cases is the expected continued occurrence of regularly distributed events beyond the reference interval. At least some IMPF-asuniversal accounts (e.g. Bonomi 1997), lack an intensional component to capture this expectation of continuation. ${ }^{5}$

\section{A Partition-Based Analysis}

The IMPF-as-universal approach is confronted with the same set of problems that any universal analysis of genericity is confronted with - the problems of determining restriction domains, accounting for exceptions, and intensional behavior. In order to have some explanatory value, the IMPF-as-universal analysis requires a contentful theory regarding the nature of the quantifier restriction. Specifically, the theory needs to account for exception tolerance and to take the intensional aspect of IMPF-marked sentences into consideration. The analysis proposed here aims to capture the intuition that characterizing sentences are statements about the expected continuation of a regular distribution of events across time.

\footnotetext{
${ }^{4} \mathrm{An}$ obvious solution to this is to weaken universal quantification to generic quantification of the sort contributed by GEN (e.g. Lenci \& Bertinetto 2000); however that considerably weakens the explanatory force of the IMPF-as-universal analysis.

${ }^{5}$ Lenci \& Bertinetto (2000) present a modal intensional IMPF-as-universal account relying on a stereotypical ordering source. The analysis proposed in this paper expresses the intensional aspect of imperfectivity making use of the branching time framework.
} 


\subsection{The Setup}

The formal framework is based on the branching time semantics proposed in Thomason $(1970,1984)$. A treelike frame consists of a pair $\langle\mathscr{T}, \prec\rangle$, where $\mathscr{T}$ is a nonempty set of times with dense ordering and $\prec$ is a transitive tree-like relation on $\mathscr{T}$ such that for all $t, u, v \in \mathscr{T}$ if $u \prec t$ and $v \prec t$, then either $u \prec v$ or $v \prec u$ if $u \neq v$. A history (or maximal chain) on $\mathscr{T}$ is a subset $h$ of $\mathscr{T}$ such that (a) for all $t, u \in h$, if $t \neq u$, then $t \prec u$ or $u \prec t$, and (b) if $g$ is any subset of $\mathscr{T}$ such that for all $t, u \in g$, if $t \neq u$, then $t \prec u$ or $u \prec t$, then $g=h$ if $h \subseteq g$. For any $t \in \mathscr{T}, H_{t}$ is the set of histories containing $t$.

In addition to the set of times $\mathscr{T}$, the ontology contains intervals and sorted eventualities. An interval $i$ is a subset of $\mathscr{T}$ such that (1) $i$ is a proper subset of some history $h$ in $\mathscr{T}$, and (2) for all $t_{1}, t_{2}, t_{3} \in h$, if $t_{1}, t_{3} \in i$ and $t_{1} \prec t_{2} \prec t_{3}$ then $t_{2} \in i$ (Dowty, 1977: 64). Let $\mathscr{I}$ be a domain of non-null intervals (with points as a special case), partially ordered by the relation of temporal precedence $\prec$ and by the subinterval relation $\subseteq . i, j, k \ldots$ are variables over $\mathscr{I}$ and $H_{i}$ is the set of histories containing $i$. The function $I n r$ assigns to each $i \in \mathscr{I}$ a proper subset of the histories containing $i-H_{i_{i n r}}$, which are the inertia futures of $i$ (Dowty, 1979: 152). ${ }^{6}$

$$
\begin{aligned}
& \text { Inertia futures } \\
& \text { Inr }=f: I \rightarrow \wp(H) \\
& i \mapsto H_{i_{\text {inr }}} \subset H_{i}
\end{aligned}
$$

For any interval $i$ a subset of $\mathscr{T}$, a partition of $i$ is the set of the non-empty, mutually exclusive, and collectively exhaustive subsets of $i$. The notion of a regular partition of $i$ is defined in (7).

\section{Regular partition}

$\mathscr{R}_{i}$ is a regular partition of $i$ if $\mathscr{R}_{i}$ is a set of intervals $\{j, k \ldots n\}$ such that
a. $\bigcup\{j, k \ldots n\}=i$
b. $\forall j, k \in \mathscr{R}_{i} \rightarrow j \cap k=\emptyset$ if $j \neq k$
c. $\forall j, k \in \mathscr{R}_{i} \rightarrow \mu(j)=\mu(k)$ (where $\mu(x)$ stands for the Lebesgue measure of $x)^{7}$

\footnotetext{
${ }^{6}$ Dowty $(1977,1979)$ introduces the notions of inertia worlds and inertia futures as a means to access the set of worlds/histories that are indistinguishable from each other up until the reference interval and continue past this interval in ways that are compatible with the normal course of events. Much literature on the Imperfective Paradox has focused on refining Dowty's notion of inertia, particularly relativizing it to the predicate and event under question (Landman 1992; Portner 1998). It is not within the scope of this paper to contribute to these refinements to the concept of inertial futures. Both the event-in-progress and the characterizing uses of IMPF depend on the future behaving in ways predictable from the past and the present. Inr is intended to be a placeholder function that allows us restrict our attention to histories that meet this predictability requirement.
} 
For any $\mathscr{R}_{i}$, each of its subsets will have the same measure and this measure will be referred to by the term partition-measure. Intuitively, a regular partition of $i$ is a set of non-overlapping chunks of time of equal length partitioning $i$, a set against which predicate-instantiation may be evaluated with respect to regular distribution in time.

$\mathscr{E}$ is a non-null domain of events. The temporal trace function $\tau$ from $\mathscr{E}$ to $\mathscr{I}$ gives the run time of an event. Lexically episodic predicates denote sets of events. Lexically stative predicates denote predicates over intervals. Sentence radicals are predicates built from basic eventive or stative predicates with their individual arguments saturated (somewhat corresponding to the VP-level assuming VPinternal subjects). Aspectual modifiers such as negation, frequency and Q-adverbs, and quantified PPs apply to basic eventive and stative predicates to yield predicates of intervals. Aspectual operators like the imperfective and perfective may either apply to the denotations of sentence radicals or to the predicates of intervals returned by aspectual modifiers. They map properties of events/intervals to sets of intervals relative to which these predicates are instantiated and this may involve existential quantification over the Davidsonian event variable. In a branching time ontology, instantiation is relative to a time and a history, The instantiation of properties at a time and a history is specified here in terms of the colncidence relation defined as in (8). In words, $P$ is in a coincidence relation with $i$ and $h$ if $P$ holds within $i$ or at a superinterval of $i$ and the time at which $P$ holds is a subset of $h$.

$$
\operatorname{COIN}(P, i, h)= \begin{cases}\exists e[P(e) \wedge \tau(e) \circ i \wedge \tau(e) \subset h] & \text { if } P \subseteq \mathscr{E} \\ P(i) \wedge i \subset h & \text { if } P \subseteq \mathscr{I}\end{cases}
$$

Tense operators are functions that map predicates of eventualities or intervals to propositions, instantiating these properties in time.

\subsection{The Meaning of IMPF}

The IMPF operator is defined in (9). According to (9), IMPF applies to a predicate (of eventualities or intervals) $P$ to yield a predicate of intervals $i$ such that (a) every inertia future of $i$ contains an interval $j$ (where $i$ is a non-final subinterval of $j$ ) and (b) every cell $k$ of a contextually determined regular partition of $j, \mathscr{R}_{j}{ }^{c}$, CoINcides with $P$. A contextually determined regular partition is a regular partition where the partition measure is anaphoric on the context.

$$
\text { IMPF: } \lambda P \lambda i \forall h\left[h \in H_{i_{i n r}} \rightarrow \exists j\left[i \subset_{n f} j \subset h \wedge \forall k\left[k \in \mathscr{R}_{j}{ }^{c} \rightarrow \operatorname{COIN}(P, k, h)\right]\right]\right]
$$

\footnotetext{
${ }^{7}$ The Lebesgue measure is the standard way of assigning a length, area, or volume to subsets of Euclidean space. Intervals are a proper subset of the Lebesgue measurable subsets of the real number line.
} 
The intuition with characterizing sentences is that they describe the expected continuation of a regular distribution of events across time. The introduction of inertia futures (histories) into the meaning of IMPF allows us to formalize the expected continuation intuition. The universal quantifier quantifies not over the subintervals of the reference interval, but over the subintervals of future-extending superintervals of the reference interval.

The intuition about regular distribution in time is expressed via the notion of a regular partition. The actual frequency of events that is necessary to evaluate a pattern of episodes as regular varies from context to context, and is best treated as a function of context. Treating the partition-measure (the object that generates the restriction set) as a free variable whose value is provided by context, captures this variability. The predicate in the scope of IMPF coincides with every cell of this context-determined partition.

(11) provides a step-by-step derivation demonstrating how the proposed meaning for IMPF combines with other semantic components in order to build up the meaning of IMPF-marked sentences. Let us assume that the Gujarati Imperfective realizes IMPF as given in $(10){ }^{8}$

$$
\begin{aligned}
& \llbracket \text {-e } \rrbracket \\
& =\lambda P \lambda i \forall h\left[h \in H_{i_{i n r}} \rightarrow \exists j\left[i \subset{ }_{n f} j \subset h \wedge \forall k\left[k \in \mathscr{R}_{j}{ }^{c} \rightarrow \operatorname{COIN}(P, k, h)\right]\right]\right]
\end{aligned}
$$

The logical form for (3a) is in (11c). According to (11c), (3a) is true iff there is an $i$ that contains now and every inertia future of $i$ contains a $j$, s.t. $i \subset{ }_{n f} j \subset h$, s.t. every $k \in \mathscr{R}_{j}^{c}$ overlaps with the time of an event of Niśā making bread.

$$
\begin{aligned}
& \text { a. PRES (IMPF }(\lambda e[\text { Niśā-make-bread }(e)])) \\
& \text { b. PRES }\left(\lambda P \lambda i \forall h\left[h \in H_{i_{i n r}} \rightarrow \exists j\left[i \subseteq{ }_{n f} j \subset h \wedge \forall k\left[k \in \mathscr{R}_{j}{ }^{c} \rightarrow \operatorname{COIN}(P, k, h)\right]\right]\right]\right. \\
& (\lambda e[\operatorname{Niśā-make-bread}(e)])) \\
& =\operatorname{PRES}\left(\lambda i \forall h \left[h \in H _ { i _ { i n r } } \rightarrow \exists j \left[i \subseteq { } _ { n f } j \subset h \wedge \forall k \left[k \in \mathscr{R}_{j}{ }^{c} \rightarrow \operatorname{COIN}(\lambda e[\text { Niśā- }\right.\right.\right.\right. \\
& \operatorname{make-bread}(e)], k, h)]]]) \\
& =\operatorname{PRES}\left(\lambda i \forall h \left[h \in H _ { i _ { i n r } } \rightarrow \exists j \left[i \subseteq { } _ { n f } j \subset h \wedge \forall k \left[k \in \mathscr{R}_{j}{ }^{c} \rightarrow \exists e[\text { Niśā-make- }\right.\right.\right.\right. \\
& \operatorname{bread}(e) \wedge \tau(e) \circ k \wedge \tau(e) \subset h]]]]) \\
& \text { c. } \exists i\left[\text { now } \subseteq i \wedge \forall h \left[h \in H _ { i _ { i n r } } \rightarrow \exists j \left[i \subseteq { } _ { n f } j \subset h \wedge \forall k \left[k \in \mathscr{R}_{j}{ }^{c} \rightarrow \exists e[\text { Niśā- }\right.\right.\right.\right. \\
& \operatorname{make-bread}(e) \wedge \tau(e) \circ k \wedge \tau(e) \subset h]]]]]
\end{aligned}
$$

Remember that the problem with an IMPF-as universal analysis that relies on an unexplicated reference to contextual relevance is that it is not always clear what the restriction for the universal quantifier is. For a sentence like (3b) uttered with neutral intonation, the set of intervals in which Niśā makes something, or the set of intervals in which someone makes bread do not seem provide the right

\footnotetext{
${ }^{8}$ The Gujarati Imperfective paradigm is represented here by the expression $-e$, which is the third person singular affix.
} 
restriction. In the analysis proposed here, the quantifier restriction is taken to be a regular partition rather than some implicit set of events such as those involving Niśā cooking. The sentence makes an assertion about the regular distribution of bread-making events in time, not an exclusive correlation between, for instance, cooking events and bread-making events. The sentence may be judged true even if Niśa makes bread outside the kitchen, or if she makes pasta instead of bread, on some days, as long as there is a salient partition whose every member overlaps with a bread-making event by Niśa. The context determines the restriction in a principled way; it does not provide a predicate, nor does it rule out those members of a restriction set to which a generalization does not apply as being irrelevant to the quantification. It only provides the partition-measure, a measure of length which serves to draw a partition which constitutes the restriction set.

\section{Deriving Imperfective Readings}

\subsection{The Characterizing Reading}

The characterizing reading of IMPF-marked sentences arises when the partitionmeasure is set to a relatively large length (e.g. a few days, weeks, fortnights, months, the value depending on context). (3a) can be an answer to different explicit or implicit questions. For instance, if the discourse context has to do with Niśâ's preferred dinner item, the partition-measure is likely to be set to a few days, giving rise to an inference that the bread-making is frequent. On the other hand, if the discourse context has to do with whether Niśâ's cooking abilities, (3a) may be uttered to convey the information that Niśā does have some bread-making abilities, though the bread-making events might be few and far between. In such a context, the partition-measure is correspondingly large.

It has been pointed out that characterizing sentences are associated with at least two kinds of generic reading - one a universal habitual reading and the other a weaker existential dispositional one (Lawler 1973, Dahl 1975, Krifka et al 1995). For example, an IMPF-marked characterizing sentence like (12) can be read in two ways. On the habitual reading, it means that Rām eats meat with a high degree of frequency; in fact, most of the times that he eats food, the food involves meat. On the dispositional reading, the sentence says that meat is not a kind of food that Rām does not eat.

$$
\begin{aligned}
& \text { rām mā̄̄āhārī anna khā-e che } \\
& \text { rām.NOM non-vegetarian food.NOM eat-IMPF.M.SG PRES.3.SG } \\
& \text { Rām eats meat (non-vegetarian food). }
\end{aligned}
$$

In contrast to Lawler's proposal to account for the contrast by employing 
two hidden generic operators, Krifka et al retain a single universal meaning for the generic operator and account for the readings via different partitions of the semantic material. The partitioning for the two readings of (12) is in (13).

a. GEN $[\mathrm{x}, \mathrm{y}, \mathrm{s} ;](\mathrm{x}=\mathbf{R} \overline{\mathbf{a} m} \& \mathrm{x}$ eats $\mathrm{y}$ in $\mathrm{s} ; \mathrm{y}$ is meat $)$

b. GEN $[x, y, s ;](x=R a \bar{m} \& y$ is meat \& $x$ in $s \& y$ in $s ; x$ eats $y$ in $s)$

Focus, expressed through accent placement (on the object, which corresponds to (13a) or on the verb, which corresponds to (13b)), justifies the distinct structures associated with the two readings. (13b) says that if a situation contains Rām and meat, it is generally a situation in which Rām eats the meat. However, on the dispositional reading, (12) is likely to be evaluated as true even if Rām normally does not eat meat when it is available (because he is partial to vegetarian food, which he habitually eats), as long as some of the times he does eat it.

A possible account for this contrast within the proposal made here can be sketched out in the following way. The habitual and the dispositional generic readings necessarily differ with respect to the absolute frequency of the events denoted by the sentence radical, within some larger interval. The habitual reading implies a high frequency instantiation of such events, while the dispositional reading implies a sparser frequency of such events. This difference can be naturally captured by the context-induced variability of the partition-measure. If the discourse context is concerned with whether Rām has the habit of eating meat, the context provides a partition of the appropriately short measure (e.g. a measure of the length of a couple of days) and every partition cell is expected to overlap with a meat-eating event. On the other hand, if the context is concerned with whether Rām objects to eating meat or not, we might consider a partition measure of much larger length (e.g a measure of the length of a year, or even longer), and each cell in such a partition is expected to overlap with a meat-eating event.

Notice that on the dispositional reading, there is no requirement that there be a meat-eating event by Rām within the actually realized part of the interval under consideration. The partition-measure may be much longer than the actual interval stretching from the left boundary of the reference time until utterance time. Imagine a scenario in which Rām, who has always been an avowed vegetarian, gives up his resolve and has decided to not always reject meat when confronted with it. This happened last week but he has not yet had the opportunity to implement his changed attitude. Nevertheless, (12) is true today since Rām's attitude supports the possibility of meat-eating events to occur with regularity (though probably with limited frequency) in the future. 


\subsection{The Event-in-Progress Reading}

The intuition for the event-in-progress reading is that the reference interval itself is understood as being a subinterval of a larger interval within which the predicate in the scope of the imperfective operator holds (hence the familiar Reichenbachian $\mathrm{R}$ $\subset \mathrm{E}$ value for the imperfective). This intuition can be expressed with the proposed meaning for IMPF.

On the current analysis, IMPF-marked sentences exhibit the event-in-progress reading when the partition-measure is chosen from the set of infinitesimals; i.e when the measure is set to an infinitesimally small length. $\mathscr{R}_{i}{ }^{i n f}$ (I will call this an $I$ partition) is the set of mutually exclusive and collectively exhaustive subsets of an interval $i$ of infinitesimally small measure. ${ }^{9}$ If every such subset of $i$ CoINcides with a predicate $P$ that IMPF applies to, then $i$ itself coincides with $P$. Specifically, for a predicate of eventualities $P, i$ is guaranteed to be a subinterval of the time of an event $e$ instantiating $P$. The main attractive consequence of this move is that it allows us to uniformly retain partitions as the restriction set for the universal quantifier associated with IMPF. (15) derives (14), (repeated from (1a)).

niśā rasợā-mā roṭli banāv-e ch-e

N.NOM kitchen-LOC bread.NOM make-IMPF.3.SG PRES-3.SG

'Niśa is making bread in the kitchen (right now).' event-in-progress

$\operatorname{IMPF}(\lambda e[$ Niśā-make-bread-in-the-kitchen $(e))$

$=\lambda P \lambda i \forall h\left[h \in H_{i_{\text {inr }}} \rightarrow \exists j\left[i \subset{ }_{n f} j \subset h \wedge \forall k\left[k \in \mathscr{R}_{j}{ }^{i n f} \rightarrow \operatorname{COIN}(P, k, h)\right]\right]\right](\lambda e$ Niśāmake-bread-in-the-kitchen $(e))$

$=\lambda i \forall h\left[h \in H_{i_{i n r}} \rightarrow \exists j\left[i \subset{ }_{n f} j \subset h \wedge \forall k\left[k \in \mathscr{R}_{j}{ }^{\text {inf }} \rightarrow \exists e[\right.\right.\right.$ Niśā-make-breadin-the-kitchen $(e) \wedge \tau(e) \circ k \wedge \tau(e) \subset h]]]]$

According to (15), (14) is true at an interval $i$ iff $i$ is a non-final subinterval of a larger interval $j$ (in every inertial history), such that every subinterval that is in the I-partition of $j$ overlaps with an event $e$ of the janitor opening the door. That is, if the relation $i \subset_{n f} j \subseteq \tau(e)$ holds. If every subset $k \in \mathscr{R}_{j}{ }^{i n f}$ (where $c$ is determined to some infinitesimal measure) COINcides with a predicate $P$ that IMPF applies to, then $j$ itself coIncides with $P$. It follows that $i$ is a subinterval of the time of an event $e$ instantiating $P$.

For an accomplishment predicate of eventualities $P_{a c c}$, assuming an I-partition, $\operatorname{IMPF}\left(P_{a c c}\right)$ will yield the set of non-final subintervals of any interval at which $P_{a c c}$ holds. For an activity predicate $P_{a c t}$ (e.g. walk, run) with the subinterval property, $\operatorname{IMPF}\left(P_{a c t}\right)$ will correspond to the set of non-final intervals of any interval

\footnotetext{
${ }^{9}$ This idea of expressing the subinterval relation between the reference interval and a single event interval by allowing the partition-measure to be set at infinitesimal length is due to Mokshay Madiman from the Yale Statistics Department.
} 
such that $P_{a c t}$ also holds at each of its subintervals (above a certain granularity). The same holds for a semelfactive predicate $P_{\text {sem }}$ (e.g. knock, tap). This generates the on-going process reading for activity predicates and the iterative reading for semelfactives.

\subsection{The Continuous Reading}

The continuous reading arises with lexical stative predicates, which denote predicates over intervals. As per the definition of COINcidence, for any lexically stative predicate $P_{\text {stat }}, \operatorname{IMPF}\left(P_{\text {stat }}\right)$ will be a subset of $P_{\text {stat }}$.

$$
\operatorname{COIN}(P, i, h)= \begin{cases}\exists e[P(e) \wedge \tau(e) \circ i \wedge \tau(e) \subset h] & \text { if } P \subseteq \mathscr{E} \\ P(i) \wedge i \subset h & \text { if } P \subseteq \mathscr{I}\end{cases}
$$

According to (18), (17) is true iff there is an interval $i$ (containing now) and every inertia future $h$ through $i$ contains a $j$, s.t. $i \subseteq{ }_{n f} j$ and every $k \in \mathscr{R}_{j}{ }^{c}$ is such that Niśā lives in Navsari is true at $k$. In other words, Niśā's living in Navsari is asserted to hold throughout a superinterval of $i$.

niśā navsāri-mā rah-e ch-e

N.NOM.SG Navsari-LOC live-IMPF.3.SG PRES-3.SG

Niśā lives in Navsari.

Continuous

$\exists i\left[\right.$ now $\subseteq i \wedge \forall h\left[h \in H_{i_{\text {inr }}} \rightarrow \exists j\left[i \subseteq{ }_{n f} j \subset h \wedge \forall k\left[k \in \mathscr{R}_{j}{ }^{c} \rightarrow\right.\right.\right.$ Niśā-live-in$\operatorname{Navsari}(k) \wedge k \subset h]]]]$

\section{Typological Variation}

So far, I have shown that it is possible to assume a single meaning for IMPF and account for its characterizing, event-in-progress, and continuous readings by assuming varying measures for a context-determined regular partition. The flavors within each of these readings - strong habitual vs. weak dispositional for the characterizing reading, and non-culminated event vs. ongoing process vs. iterative readings emerge as natural consequences of further differences in the assumed partition-measure or from the lexical properties of the predicate in the scope of IMPF.

The other question that this paper is concerned with is that of idenitfying the source of variation in the crosslinguistic manifestation of imperfectivity. What distinguishes the broader category that encompasses the event-in-progress and the characterizing readings (realized by the Gujarati Imperfective), and a narrower 
category (realized by the English Progressive) that saliently exhibits the event-inprogress reading?

The current analysis can naturally make sense of this typological variation. Progressive marking realizes PROG, which is a specific version of IMPF, namely, an operator where the partition-measure is obligatorily chosen from a set of infinitesimal values. PROG differs from IMPF only in that the partition-measure for IMPF varies by context, while that for PROG is obligatorily fixed to infinitesimal length. In other words, with PROG, the restriction of the universal quantifier is constrained to be an I-partition, guaranteeing only the event-in-progress reading for a sentence containing PROG in its logical form. The two can be compared in (19).

a. PROG: $\lambda P \lambda i \forall h\left[h \in H_{i_{i n r}} \rightarrow \exists j\left[i \subset_{n f} j \subset h \wedge \forall k\left[k \in \mathscr{R}_{j} \inf \rightarrow \operatorname{COIN}(P, k, h)\right]\right]\right]$

b. IMPF: $\lambda P \lambda i \forall h\left[h \in H_{i_{i n r}} \rightarrow \exists j\left[i \subset{ }_{n f} j \subset h \wedge \forall k\left[k \in \mathscr{R}_{j}{ }^{c} \rightarrow \operatorname{COIN}(P, k, h)\right]\right]\right]$

If this is indeed an accurate characterization of the difference between the meanings of IMPF-marking and PROG-marking, then we can expect languages to vary within a four-way typology - whether they morphologically realize IMPF or PROG or neither or both. The following table gives examples of languages which fall in each of these slots.

\begin{tabular}{|l|c|c|}
\hline \multicolumn{2}{|c|}{ OPERATOR } & \\
\hline IMPF & PROG & Languages \\
\hline \hline$\emptyset$ & $\emptyset$ & German \\
\hline$\emptyset$ & $\sqrt{ }$ & English \\
\hline$\sqrt{ }$ & $\emptyset$ & Russian, Arabic \\
\hline$\sqrt{ }$ & $\sqrt{ }$ & Italian, Gujarati \\
\hline
\end{tabular}

Within this typology, a language like English realizes PROG and therefore the English Progressive is incompatible with characterizing readings. A language like Gujarati or Italian realizes IMPF, which subsumes the meaning of PROG, and therefore the Gujarati Imperfective or the Italian Imperfetto exhibit both characterizing and event-in-progress readings. We say that IMPF subsumes PROG because one of the values that the contextual free variable for the partition-measure can receive is an infinitesimal one. Gujarati and Italian contrast with a language like Arabic in that these languages realize PROG morphologically via periphrastic devices in addition to realizing IMPF.

\subsection{The Progressive and Stativity}

Although the cross-linguistic picture of the relation between IMPF and PROG is initially appealing, there remain two outstanding problems for taking the meaning 
of the English Progressive to be PROG as defined in (19a). Specifically, we are confronted with two uses of the English Progressive that do not follow from this meaning. The first has to do with the the deteriorated acceptability (in most contexts) of individual-level statives (e.g. know, own, love) with progressive marking as in (21). The second has to do with the perfect acceptability of habitual readings for episodic predicates in the scope of the Progressive, as in (22).

(21) a. ?John is owning three houses.

b. ?Mary was knowing the answer.

a. John is baking baguettes (these days).

b. Mary was jogging five miles (last year).

Individual-level stative predicates have the subinterval property; i.e. they hold at every subinterval of any interval they hold at. Consequently, if a predicate like Mary-know-the-answer holds at an interval $i$ and is expected to hold at a superinterval $j$ of $i$, then it will automatically hold in every $k \in \mathscr{R}_{j}{ }^{i n f}$. If PROG is the meaning of the English Progressive, there is no explanation for this incompatibility.

With the habitual reading, the problem is slightly different. A sentence like (22a) crucially does not assert of an interval $i$ (more specifically of a futureextending superinterval $j$ of $i$ ) that every $k \in \mathscr{R}_{j}{ }^{i n f}$ COINcides with an event of John baking baguettes. That would require John to be at the oven or the kitchen counter twenty-four hours a day throughout the interval that these days introduces. That is certainly not the condition for evaluating the truth of (22a).

The incompatibility of statives with the Progressive has been argued to have a pragmatic source (Taylor 1977; Dowty 1979). The basic idea is that the function of the Progressive is to stativize non-stative predicates. Applying the Progressive to stative predicates is semantically vacuous since they already are stative (have the subinterval property) and this has led to a pragmatic restriction on stative verbs with Progressive marking. If stativity of the predicate in the scope of the Progressive is the underlying reason for incompatibility, then it is not clear why habitual predicates (22), which have been taken to be stative, are perfectly acceptable in the Progressive. Further, as Dowty notes in his discussion of this restriction, that the incompatibility has to do with semantic considerations that go beyond the subinterval property.

Consideration of many such examples leads to the conclusion that the progressive is acceptable with these [stative] verbs just to the degree that the subject denotes a moveable object, or to be more exact, an object that has recently moved, might be expected to move in the near future, or might possibly have moved in a slightly different situation. (Dowty 1979: 175) 
A unified account of the crosslinguistic variation between Imperfective marking and Progressive marking must provide an account of the entire range of distribution of Progressive marking and not only the salient event-in-progress reading. Such an analysis is beyond the scope of this paper but I refer the reader to Deo (2009b) where I offer a detailed proposal addressing this question.

\section{Conclusion}

The goal of this paper was to provide an account of the event-in-progress, characterizing, and continuous readings of IMPF-marked sentences while retaining a single meaning for IMPF-marking. I demonstrated that it is possible to maintain universal force for IMPF, as long as we provide a explicit proposal for the restriction of the universal quantifier. This restriction should be taken to be a regular partition over a superinterval of the reference interval, where the partition-measure is a contextually determined variable. The difference between IMPF and PROG (as realized in English) was proposed to lie in whether the partition-measure can be contextually determined or whether it is constrained to have infinitesimal value. While this offers an account of the event-in-progress reading of the English Progressive, it was observed to be inadequate in accounting for the availability of the habitual reading and the observed incompatibility of the progressive with certain stative predicates.

\section{References}

Bonomi, Andrea: 1997, 'Aspect, quantification and when-clauses in Italian', Linguistics and Philosophy 20, 469-514.

Bybee, Joan, Revere Perkins, and William Pagliuca: 1994, The Evolution of Grammar. Tense, Aspect, and Modality in the Languages of the World. The University of Chicago, Chicago.

Cipria, Alicia and Craige Roberts: 2000, 'Spanish Imperfecto and Pretérito: Truth conditions and aktionsart effects in a situation semantics', Natural Language Semantics 8, 297-347.

Comrie, Bernard: 1976, Aspect: An Introduction to the Study of Verbal Aspect and Related Problems. Cambridge University Press.

Dahl, Östen: 1975, 'On Generics', in E. Keenan (ed.), Formal Semantics of Natural Language, 99-111. Cambridge University Press, Cambridge.

Dahl, Östen: 1985, Tense and Aspect Systems. Basil Blackwell, Oxford.

Deo, Ashwini: 2006, Tense and Aspect in Indo-Aryan Languages: Variation and Diachrony, Doctoral Dissertation, Stanford University.

Deo, Ashwini: 2009a, 'Temporal genericity and the contribution of imperfective 
marking ', in Proceedings of the 10th Symposium on Logic and Language, August 26-29 2009, Balatonszemes, Hungary.

Deo, Ashwini: 2009b, 'Unifying the imperfective and the progressive: partitions as quanticational domains '. ms, Yale University.

Dowty, David: 1977, 'Toward a semantic analysis of verb aspect and the English 'imperfective' progressive', Linguistics and Philosophy 1, 45-77.

Dowty, David: 1979, Word Meaning and Montague Grammar. Kluver Academic Publishers, Dordrecht.

von Fintel, Kai: 1995, ‘A minimal theory of adverbial quantification', in B. Partee and H. Kamp (eds.), Context-dependence in the Analysis of Linguistic Meaning, Proceedings of the workshops in Prague and Bad Teinach, Vol. 1, 153-93.

Krifka, Manfred, Francis J. Pelletier, Greg Carlson, Alice ter Meulen, Gennaro Chierchia, and Godehard Link: 1995, 'Genericity: An Introduction', in The Generic Book, 1-124. The University of Chicago Press, Chicagodelfit,.

Lawler, John M.: 1973, Studies in English Generics, Doctoral Dissertation, University of Michigan, Ann Arbor.

Lenci, Alessandro and Pier Marco Bertinetto: 2000, 'Aspect, adverbs, and events: habituality vs. perfectivity', in J. Higginbotham, F. Pianesi, and A. Varzi (eds.), Speaking of Events. Oxford University Press, USA.

Menendez-Benito, Paula: 2002, 'Aspect and adverbial quantification in Spanish', in NELS Proceedings, Vol. 32, 365-382.

Newton, Brian: 1979, 'Scenarios, modality, and verbal Aspect in Modern Greek.', Language 55, 139-67.

Ryding, Karin C.: 2005, A Reference Grammar of Modern Standard Arabic. Cambridge University Press, Cambridge.

Taylor, Barry: 1977, 'Tense and continuity', Linguistics and philosophy 1, 199220.

Thomason, Richmond H.: 1970, 'Indeterminist time and truth-value gaps', Theoria 36, 264-281.

Thomason, Richmond H.: 1984, 'Combinations of tense and modality', Handbook of Philosophical Logic 2, 135-165.

Tröbs, Holger: 2004, 'Progressive and habitual aspects in Central Mande', Lingua 114, 125-163. 